Article

\title{
Superinjection of Holes in Homojunction Diodes Based on Wide-Bandgap Semiconductors
}

\author{
Igor A. Khramtsov(1) and Dmitry Yu. Fedyanin *-1] \\ Laboratory of Nanooptics and Plasmonics, Moscow Institute of Physics and Technology, Dolgoprudny 141700, \\ Russia; khramtsov@phystech.edu \\ * Correspondence: dmitry.fedyanin@phystech.edu
}

Received: 20 April 2019; Accepted: 17 June 2019; Published: 19 June 2019

check for updates

\begin{abstract}
Electrically driven light sources are essential in a wide range of applications, from indication and display technologies to high-speed data communication and quantum information processing. Wide-bandgap semiconductors promise to advance solid-state lighting by delivering novel light sources. However, electrical pumping of these devices is still a challenging problem. Many wide-bandgap semiconductor materials, such as $\mathrm{SiC}, \mathrm{GaN}, \mathrm{AlN}, \mathrm{ZnS}$, and $\mathrm{Ga}_{2} \mathrm{O}_{3}$, can be easily n-type doped, but their efficient p-type doping is extremely difficult. The lack of holes due to the high activation energy of acceptors greatly limits the performance and practical applicability of wide-bandgap semiconductor devices. Here, we study a novel effect which allows homojunction semiconductor devices, such as p-i-n diodes, to operate well above the limit imposed by doping of the p-type material. Using a rigorous numerical approach, we show that the density of injected holes can exceed the density of holes in the p-type injection layer by up to four orders of magnitude depending on the semiconductor material, dopant, and temperature, which gives the possibility to significantly overcome the doping problem. We present a clear physical explanation of this unexpected feature of wide-bandgap semiconductor $\mathrm{p}-\mathrm{i}-\mathrm{n}$ diodes and closely examine it in $4 \mathrm{H}-\mathrm{SiC}, 3 \mathrm{C}-\mathrm{SiC}, \mathrm{AlN}$, and $\mathrm{ZnS}$ structures. The predicted effect can be exploited to develop bright-light-emitting devices, especially electrically driven nonclassical light sources based on color centers in $\mathrm{SiC}, \mathrm{AlN}, \mathrm{ZnO}$, and other wide-bandgap semiconductors.
\end{abstract}

Keywords: superinjection in homojunction diodes; silicon carbide; zinc sulfide; aluminum nitride; light-emitting diodes; single-photon sources

\section{Introduction}

The possibility to create a high density of nonequilibrium charge carriers in the active region of semiconductor optoelectronic devices is essential for a wide range of optoelectronic applications, from light emitting diodes (LEDs) [1] and injection lasers [2,3] to electro-optic modulators [4,5] and recently emerged single-photon sources [6-8]. The higher the carrier density, the better the performance achieved. In most devices, the excess carriers are injected to the active region of the device from the heavily doped electron-rich n-type layers and hole-rich p-type layers, respectively [9]. However, wide-bandgap semiconductors are quite unique materials, which are at the interface between conventional semiconductors and insulators. They can demonstrate both n-type and p-type conductivity, but typically suffer from the lack of either electrons or holes due to the extremely high activation energies of dopants and nonzero compensation of donors (acceptors) by acceptor-type (donor-type) impurities [10-14]. Due to this problem, the density of free carriers in the n-type or p-type injection layer can be many orders of magnitude lower than the donor or acceptor concentration, respectively. The failure to produce enough free carriers is very often the only reason why the 
performance of light-emitting devices based on wide-bandgap semiconductors is significantly lower than that of based on conventional semiconductor materials, such as gallium arsenide $[15,16]$.

The traditional solution to this problem is to use the superinjection effect in double heterostructures [17-19]. As illustrated in Figure 1a, high potential barriers at the heterojunctions prevent electrons and holes from escaping from the central active region of the structure. Thus, under forward bias, all carriers injected from the n-type and p-type layers are confined to the central region of the double heterostructures. The densities of injected electrons and holes can be orders of magnitude higher than in the n-type and p-type injection layers (Figure 1b) [2], which is referred to as superinjection $[17,18]$. However, to exploit the superinjection effect in the particular semiconductor, one needs an auxiliary semiconductor material which has a larger bandgap properly aligned with respect to the conduction and valence band edges of the considered semiconductor (Figure 1a) and a lattice constant equal or close to that of the considered semiconductor. However, such an auxiliary material does not exist for many wide-bandgap semiconductors (see Figure 1.9 in [20]). Therefore, only homojunction structures can often be used.

For decades, it was believed that the superinjection effect was a unique feature of semiconductor heterostructures, while the maximum density of injected electrons and holes in homojunction structures could not exceed the free carrier densities in the n-type and p-type injection layers, respectively [21]. However, recently, it was discovered that the superinjection of electrons is possible in homojunction diamond $\mathrm{p}-\mathrm{i}-\mathrm{n}$ diodes [14,22,23]: At a high forward bias voltage, a potential well for electrons is formed in the i-region of the diamond $\mathrm{p}-\mathrm{i}-\mathrm{n}$ diode in the vicinity of the $\mathrm{p}-\mathrm{i}$ junction (see Figure 1c). Free electrons injected from the n-type layer are accumulated in this potential well so that their density can exceed the electron density in the n-type injection layer by orders of magnitude [23] (Figure 1d).
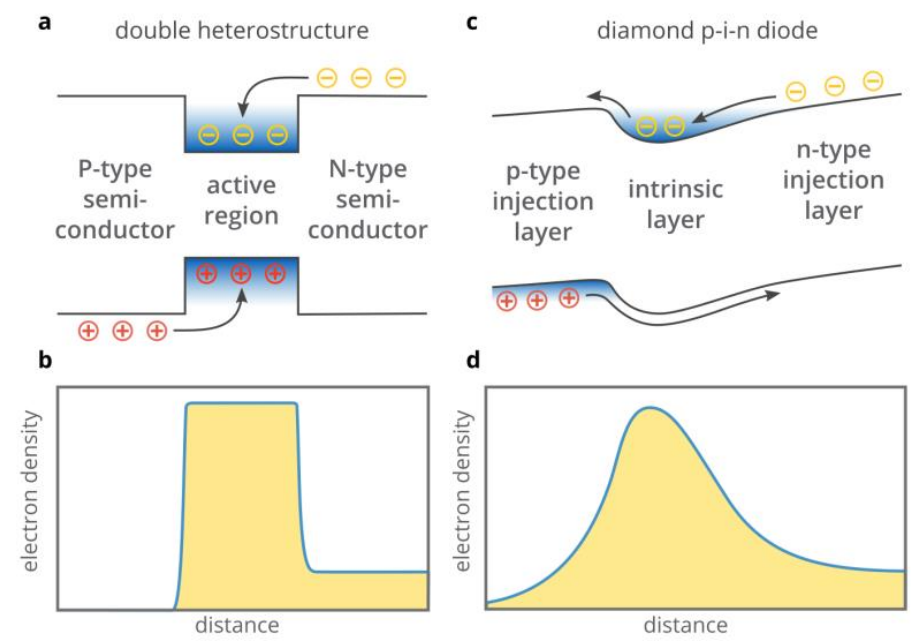

Figure 1. (a,b) Schematic illustration of electron and hole injection in a forward biased double heterostructure (a) and qualitative distribution of electron density (b). (c,d) Illustration of electron injection in a diamond p-i-n diode at a high forward bias voltage (c), and spatial distribution of electrons under these conditions (d).

In this work, using a rigorous numerical approach, we investigate for the first time the possibility to observe and exploit the superinjection effect in homojunction diodes based on wide-bandgap semiconductor materials beyond diamond. We discuss in detail how to improve the efficiency of hole injection in $\mathrm{SiC} \mathrm{p-i-n} \mathrm{diodes,} \mathrm{which} \mathrm{recently} \mathrm{demonstrated} \mathrm{great} \mathrm{promise} \mathrm{for} \mathrm{quantum} \mathrm{applications} \mathrm{as}$ single-photon sources [6,24,25]. In addition, we study the superinjection of holes in AlN and ZnS, which are promising materials for optoelectronics [26-28]. In contrast to diamond, these semiconductors do not experience noticeable problems with free electrons, but suffer from the high activation energy of acceptors. For example, AlN features an activation energy of acceptors of as high as $0.63 \mathrm{eV}$ [26], i.e., $24 k_{\mathrm{B}} T_{300 \mathrm{~K}}$, which limits the density of holes in the p-type doped material to less than $10^{10} \mathrm{~cm}^{-3}$. 
However, in this work, we show that at high forward bias voltages, it is possible to significantly overcome this doping limit using the superinjection effect in homojunction $\mathrm{p}-\mathrm{i}-\mathrm{n}$ diodes and inject far more holes into the i-region of the p-i-n diode than the doping of the p-type layer provides.

\section{Results and Discussion}

\subsection{Superinjection of Holes in $4 \mathrm{H}$-SiC Diodes}

Figure 2a shows a schematic illustration of the $4 \mathrm{H}-\mathrm{SiC}$ p-i-n diode. The n-type region of the diode is doped with nitrogen at a concentration of $N_{\mathrm{d}}=10^{18} \mathrm{~cm}^{-3}$. The activation energy of donors is $0.06 \mathrm{eV}$ [29]. The nitrogen donors are partially compensated by acceptor-type defects, and the compensation ratio is assumed to be $\eta_{\mathrm{n}}=5 \%$, which provides an electron density of $n_{\text {eqn }}=5.5 \times 10^{17} \mathrm{~cm}^{-3}$. The p-type injection layer is doped with boron. The acceptor compensation ratio is also equal to $\eta_{p}=5 \%$ [30]. The concentration of acceptors is $N_{\mathrm{a}}=10^{18} \mathrm{~cm}^{-3}$ and their activation energy equals $0.32 \mathrm{eV}$ [30], which provides a hole density of $p_{\text {eqp }}=4.3 \times 10^{14} \mathrm{~cm}^{-3}$ in equilibrium. The electron and hole mobilities in the $\mathrm{p}-, \mathrm{i}-$, and n-type regions are calculated using the theory from [31]. The size of the i-region is chosen to be $5 \mu \mathrm{m}$, which is high enough to observe the superinjection of holes at moderate currents [14]. Other parameters of the considered $4 \mathrm{H}-\mathrm{SiC}$ p-i-n diode are listed in the Supplementary Material. Using a self-consistent steady-state model, which comprises the Poisson equation, the drift-diffusion current equations and the electron and hole continuity equations to describe the charge carrier behavior in the $n-, i_{-}$, and p-type regions of the diode, we performed numerical simulations of the electron and hole transport in the p-i-n diode employing the nextnano++ software (nextnano GmbH, Munich, Germany) and our in-lab-developed simulation tool $[8,32,33]$. Both methods showed the same results.

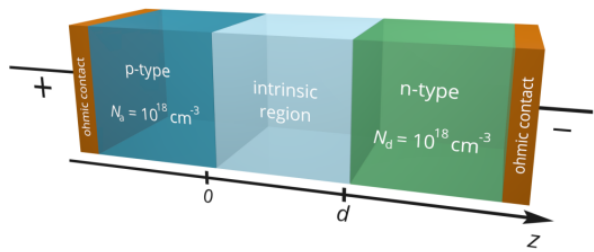

b

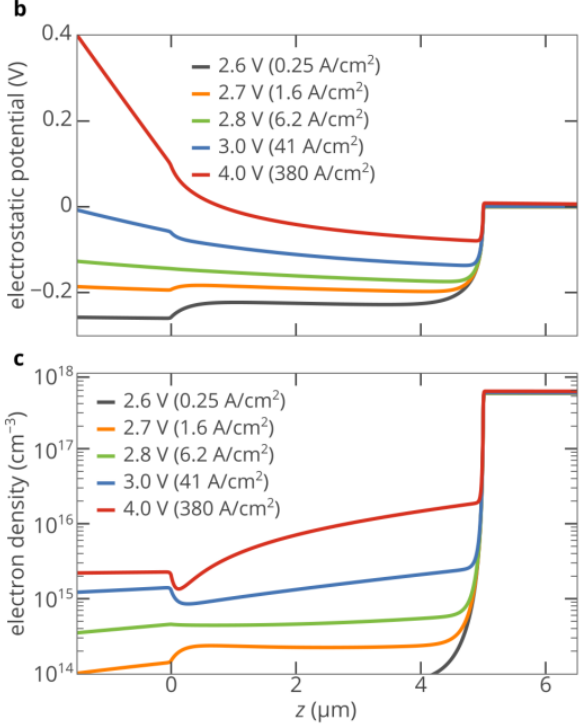

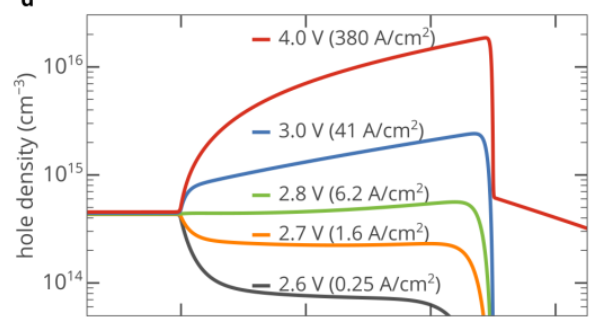

e

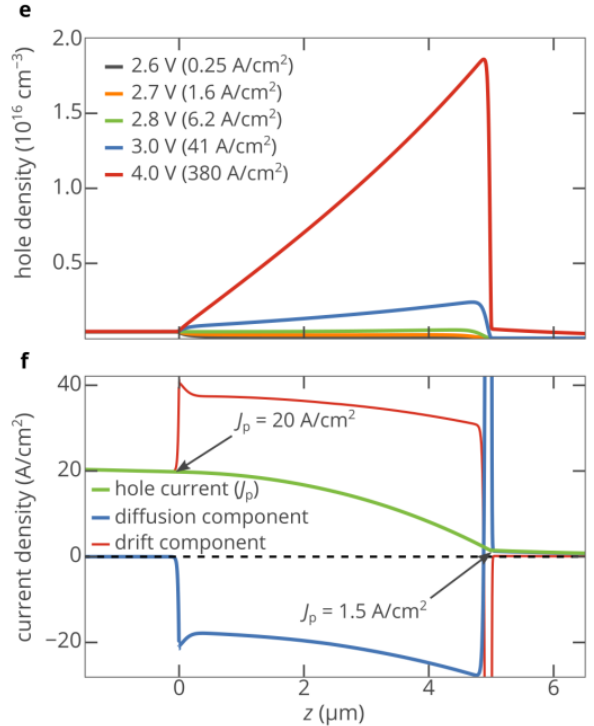

Figure 2. (a) Schematic illustration of the $4 \mathrm{H}-\mathrm{SiC}$ p-i-n diode: $d=5 \mu \mathrm{m}$; all parameters of the diode used in the simulations can be found in the Supplementary Material. (b) Electrostatic potential profile at different bias voltages. (c) Spatial distribution of electrons in the diode at different bias voltages. (d,e) Spatial distribution of holes in logarithmic (d) and linear (e) scales at different bias voltages. (f) Spatial map of the hole current and its components in the diode at $V=4.0 \mathrm{~V}\left(J=380 \mathrm{~A} / \mathrm{cm}^{2}\right)$. 
Figure 2 shows the results of the self-consistent numerical simulations of the $4 \mathrm{H}-\mathrm{SiC} \mathrm{p}$-i-n diode. It is clearly seen that at bias voltages above $2.7 \mathrm{~V}$, a potential well for holes is formed in the i-region of the diode near the $\mathrm{i}-\mathrm{n}$ junction (Figure $2 \mathrm{~b}$ ). Holes injected from the p-type layer migrate toward the n-type layer and are partially accumulated in this potential well (Figure 2d,e). The higher the bias voltage, the more holes that are collected in the well. At the same time, the potential well for holes acts as a potential barrier for electrons. Nevertheless, this barrier is located exactly at the $\mathrm{i}-\mathrm{n}$ junction (Figure 2b), and electrons easily pass over it via thermionic emission. Moreover, Figure $2 c$ shows that the electron density in the i-region near the i-n junction steadily increases with the current density, which is favorable for light-emitting devices, such as electrically pumped single-photon sources based on color centers [6,24]. Thus, at current densities above $J=6 \mathrm{~A} / \mathrm{cm}^{2}(V>2.8 \mathrm{~V})$, the maximum densities of both electrons and holes in the i-region of the p-i-n diode are found near the $\mathrm{i}-\mathrm{n}$ junction, which is counterintuitive, since one expects to find the maximum density of electrons in the proximity of the $\mathrm{i}-\mathrm{n}$ junction and the maximum density of holes in the proximity of the $\mathrm{p}-\mathrm{i}$ junction, especially in the presence of recombination. Even more surprising is the fact that at a current density of $380 \mathrm{~A} / \mathrm{cm}^{2}$, the hole density near the $\mathrm{i}-\mathrm{n}$ junction exceeds the hole density in the p-type injection layer by a factor of 44 (see Figure 2d,e).

To understand the observed superinjection of holes in the $4 \mathrm{H}-\mathrm{SiC}$ p-i-n diode, let us look at Figure $2 \mathrm{~b}$. It is clearly seen that at high forward bias voltages $(V \gtrsim 3 \mathrm{~V})$, the band bending is very strong in the p-type region, i.e., the electric field $E$ is high. At the same time, the bands are almost flat, i.e., $E \approx 0$, in the n-type region. The reason for this is that the activation energy of acceptors is much higher than the activation energy of donors, and accordingly, the density of free carriers in the n-type region is three orders of magnitude higher than in the p-type region. Therefore, at high bias voltages, the drift hole transport dominates in the p-region, while the diffusion hole transport dominates in the $\mathrm{n}$-region, which can be seen in Figure 2f. Thus, in the p-type region near the $\mathrm{p}-\mathrm{i}$ junction, the hole current density $J_{p}$ can be expressed as

$$
\left.J_{\mathrm{p}}\right|_{z=-0}=\left.\left.\left.q \mu_{\mathrm{p}}\right|_{z=-0} E\right|_{z=-0} p\right|_{z=-0},
$$

where $p$ is the hole density, $E$ is the electric field, $\mu_{\mathrm{p}}$ is the hole mobility, and $q$ is the electron charge. On the contrary, in the n-type region near the i-n junction,

$$
\left.J_{\mathrm{p}}\right|_{z=d+0} \approx-q D_{\mathrm{p}} \nabla p=\left.\left.q D_{\mathrm{p}}\right|_{z=d+0} p\right|_{z=d+0} / L_{\mathrm{p}}
$$

where $D_{\mathrm{p}}$ is the hole diffusion coefficient, $L_{\mathrm{p}}$ is the hole diffusion length in the n-type layer, and $d$ is the size of the i-region. The hole current at the $\mathrm{p}-\mathrm{i}$ junction and the hole current at the $\mathrm{i}-\mathrm{n}$ junction are connected to each other via the current continuity equation:

$$
\left.J_{\mathrm{p}}\right|_{z=d+0}=\left.J_{\mathrm{p}}\right|_{z=-0}-q \int_{0}^{d} R(z) d z,
$$

where $R(z)$ is the net recombination rate. We can rewrite this equation as

$$
\left.J_{\mathrm{p}}\right|_{z=d+0}=\left.J_{\mathrm{p}}\right|_{z=-0} / K
$$

where $K$ represents the hole current reduction factor due to recombination in the i-region. If the recombination rate is low, $K \approx 1$ and $\left.\left.J_{\mathrm{p}}\right|_{z=d+0} \approx J_{\mathrm{p}}\right|_{z=-0}$. Using Equations (1)-(4), we obtain a relation connecting the hole densities at the $\mathrm{p}-\mathrm{i}$ and $\mathrm{i}-\mathrm{n}$ junctions:

$$
\left.p\right|_{z=d+0}=\left.\left.\frac{1}{K}\left(\frac{\left.\mu_{\mathrm{p}}\right|_{z=-0}}{\left.\mu_{\mathrm{p}}\right|_{z=d+0}} \frac{q L_{\mathrm{p}}}{k_{\mathrm{B}} T}\right) E\right|_{z=-0} p\right|_{z=-0}
$$


where $k_{\mathrm{B}}$ is the Boltzmann constant and $T$ is the device temperature.

Equation (5) shows that if the electric field in the p-type layer is strong and the recombination rate in the i-region is low or moderate (i.e., $K$ is not very high), the hole density $\left.p\right|_{z=d+0}$ in the n-region in the vicinity of the $\mathrm{i}-\mathrm{n}$ junction can exceed the hole density in the p-type layer. Moreover, the hole diffusion current at the $\mathrm{i}-\mathrm{n}$ junction is positive, i.e., the gradient of the hole density at $z=d$ is negative. This means that the hole density in the i-region in the vicinity of the $i-n$ junction is even higher than $\left.p\right|_{z=d+0}$.

Figure S1 in the Supplementary Material shows that if the recombination in the i-region is negligible, i.e., $K \approx 1$, the simple Equation (5) accurately predicts that at high forward bias voltages, the hole density at the $\mathrm{i}-\mathrm{n}$ interface increases above the hole density in the p-type injection layer. If the recombination in the i-region is strong, we should take into account the bias-dependent hole current reduction factor $K$. Nevertheless, we find that at a bias voltage of $4 \mathrm{~V},\left.p\right|_{z=d+0}=7.0 \times 10^{14} \mathrm{~cm}^{-3}$, which is 1.6 times higher than the hole density in the p-type injection layer. This value agrees with that obtained in the self-consistent simulations $\left(6.0 \times 10^{14} \mathrm{~cm}^{-3}\right.$ (see Figure 2d)). The hole density in the i-region in the vicinity of the $\mathrm{i}-\mathrm{n}$ junction is even much higher, since the hole diffusion current at the $\mathrm{i}-\mathrm{n}$ interface, which is proportional to $-\nabla p$, is high and positive. Numerical simulations show that $p$ reaches $1.9 \times 10^{16} \mathrm{~cm}^{-3}$ at a distance of $120 \mathrm{~nm}$ from the $\mathrm{i}-\mathrm{n}$ junction (Figure $2 \mathrm{~d}$,e), which is 44 times higher than the hole density in the p-type injection layer.

The above explanation shows that the superinjection effect in the $4 \mathrm{H}-\mathrm{SiC} \mathrm{p}-\mathrm{i}-\mathrm{n}$ diode arises at high bias voltages due to the high contrast in the hole transport mechanisms of the p-type and n-type injection layers, which is naturally provided by the very high activation energy of acceptors. The potential well for holes near the $\mathrm{i}-\mathrm{n}$ junction, which is formed at high bias voltages and where holes are accumulated, is a consequence rather than a cause of the superinjection effect. The real reason for the superinjection effect is the requirement for the current continuity, which can be ensured only by an area with a high hole density between two regions (the p-type and n-type regions of the diode) with different (drift and diffusion) hole transport mechanisms. Therefore, the superinjection of holes can be observed in many wide-bandgap semiconductor $p$-i-n diodes that feature a similar asymmetry in the hole conduction mechanisms of the n-type and p-type layers. The key requirement for such an asymmetry is a low density of free carriers in the p-type layer and a high density of free carriers in the n-type layer.

Figure 3 shows that the maximum density of nonequilibrium holes created in the i-region in the vicinity of the i-n junction $p_{\max }$ increases with the injection current. At a current density of $15 \mathrm{kA} / \mathrm{cm}^{2}$, $p_{\max }$ reaches $2.1 \times 10^{17} \mathrm{~cm}^{-3}$, which is 500 times higher than the hole density in the p-type injection layer. Although such an injection current seems to be too high for practical applications, a significant improvement of hole injection can be achieved at current densities as low as $\sim 100 \mathrm{~A} / \mathrm{cm}^{2}$ (Figure 3).

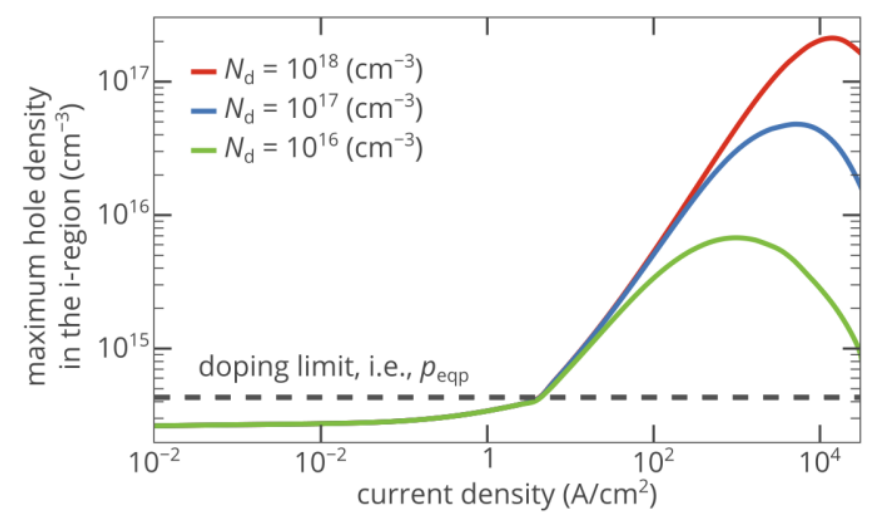

Figure 3. Dependence of the maximum hole density in the i-region of the $4 \mathrm{H}-\mathrm{SiC} \mathrm{p}-\mathrm{i}-\mathrm{n}$ diode on the injection current for different donor concentrations in the n-type injection layer. The $50 \mathrm{~nm}$-thick areas near the $\mathrm{p}-\mathrm{i}$ and $\mathrm{i}-\mathrm{n}$ junctions are ignored to avoid overestimation of the density of injected holes. 
Finally, we note that the strength of the superinjection effect decreases at extremely high bias voltages (Figure 3), since under these conditions, the drift current becomes significant even in the electron-rich n-type layer. Hence, the diode structure loses the asymmetry in the conductivity mechanisms of the n-type and p-type layer, which is required for the superinjection effect.

\subsection{Impact of Doping of the n-type Injection Layer}

As discussed in the previous section, the superinjection effect in the p-i-n diode arises due to the high contrast in the conductivity mechanisms of the p-type and n-type layers. If at a fixed bias voltage, the density of free carriers in the n-type injection layer decreases, the electric field in the n-type layer increases, which directly follows from the Poisson equation. Accordingly, the ratio of the drift hole current to the diffusion hole current increases at the $\mathrm{i}-\mathrm{n}$ junction. Since the asymmetry in conduction mechanisms of the n-type and p-type injection layers reduces, the strength of the superinjection effect decreases, which can be seen in Figure 3.

\subsection{Impact of the Activation Energy of Acceptors}

The activation energies of donors and acceptors are critical parameters that can strongly affect the efficiency of hole injection. Since the activation energy of donors in $4 \mathrm{H}-\mathrm{SiC}$ is much lower than the activation energy of acceptors [30], we focus only on the latter. The density of holes in the p-type layer rapidly decreases as the activation energy of acceptors $E_{\mathrm{A}}$ increases, especially at a nonzero acceptor compensation ratio [34]:

$$
p_{\text {eqp }}=\frac{\eta_{\mathrm{p}} N_{\mathrm{a}}+p_{1}}{2}\left(\sqrt{1+\frac{4 N_{\mathrm{a}}\left(1-\eta_{\mathrm{p}}\right) p_{1}}{\left(\eta_{\mathrm{p}} N_{\mathrm{a}}+p_{1}\right)^{2}}}-1\right),
$$

where

$$
p_{1}=\frac{1}{g_{\mathrm{A}}} N_{\mathrm{v}} \exp \left(-\frac{E_{\mathrm{A}}}{k_{\mathrm{B}} T}\right) .
$$

Here, $N_{\mathrm{a}}$ is the acceptor concentration, $g_{\mathrm{A}}$ is the degeneracy factor of the acceptor level, and $N_{\mathrm{v}}$ is the effective density of states in the valence band. Figure 4a shows the dependence of the hole density on the activation energy of acceptors for an acceptor compensation ratio of $\eta_{\mathrm{p}}=5 \%$. At $E_{\mathrm{A}}=0$, the density of holes in the p-type region $p_{\text {eqp }}$ is as high $8.2 \times 10^{17} \mathrm{~cm}^{-3}$. However, at $E_{\mathrm{A}}=0.23 \mathrm{eV}$, $p_{\text {eqp }}$ decreases by a factor of 70 , to $1.14 \times 10^{16} \mathrm{~cm}^{-3}$. This activation energy corresponds to aluminum, which is the most shallow acceptor in $4 \mathrm{H}-\mathrm{SiC}$ [30]. At $E_{\mathrm{A}}=0.5 \mathrm{eV}, p_{\text {eqp }}$ is as low as $4.1 \times 10^{11} \mathrm{~cm}^{-3}$, i.e., six orders of magnitude lower than the concentration of acceptors. However, the lower the density of holes in the p-type layer, the higher the asymmetry in the conduction properties of the p-type and n-type regions, and therefore the stronger the superinjection effect in the p-i-n diode.

Figure $4 \mathrm{~b}$ shows that the superinjection effect is very weak and can hardly be detected if the activation energy of acceptors is lower than $0.15 \mathrm{eV}$. If $4 \mathrm{H}-\mathrm{SiC}$ is doped with aluminum $\left(E_{\mathrm{A}}=0.23 \mathrm{eV}\right)$, the maximum density of injected holes can exceed the hole density in the p-type layer by more than tenfold (see Figure $4 \mathrm{~b}$ ). As the activation energy of acceptors further increases, the superinjection effect strengthens (Figure $4 \mathrm{~b}-\mathrm{d}$ ). Moreover, it can be achieved at lower currents (Figure $4 \mathrm{~b}$ ). Figure 5 shows that although the activation energy of boron $(0.32 \mathrm{eV})$ is significantly higher than the activation energy of aluminum $(0.23 \mathrm{eV})$, at room temperature and current densities above $500 \mathrm{~A} / \mathrm{cm}^{2}$, the superinjection effect allows the boron-doped p-type layer to inject roughly the same density of holes as the aluminum-doped layer can inject at the same current density. At $E_{\mathrm{A}}>0.4 \mathrm{eV}$, a greatly improved hole injection can be obtained at current densities below $10 \mathrm{~A} / \mathrm{cm}^{2}$, which is particularly interesting for practical applications. At higher injection currents, the strength of the superinjection effect can exceed 1000. However, we should note that such a very high improvement of the hole injection efficiency is achieved only in a relatively narrow region near the $i-n$ junction (see Figure $4 d$ and red 
curve in Figure 4c). Nevertheless, this feature could be advantageous for some applications, such as single-photon sources based on color centers [6,24].
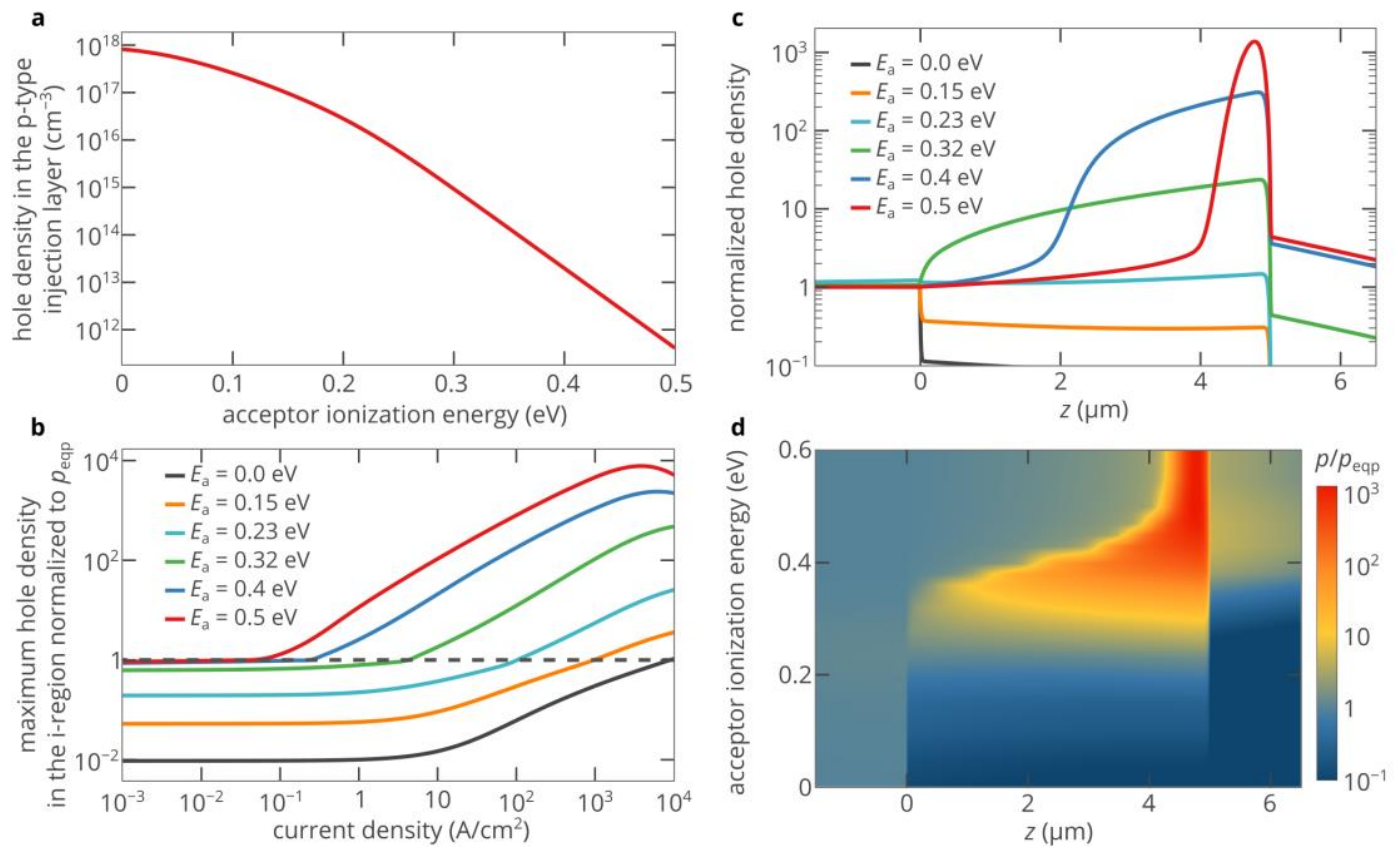

Figure 4. (a) Dependence of the hole density in the p-type injection layer on the acceptor ionization energy. The acceptor compensation ratio is equal to $5 \%$. (b) Dependence of the maximum hole density in the i-region of the $4 \mathrm{H}-\mathrm{SiC} \mathrm{p}$-i-n diode, which is normalized to the density of holes in the p-type layer $p_{\text {eqp }}$, on the injection current for different activation energies of acceptors in the p-type injection layer. The $50 \mathrm{~nm}$-thick areas near the $\mathrm{p}-\mathrm{i}$ and $\mathrm{i}-\mathrm{n}$ junctions are ignored to avoid overestimation of the density of injected holes. (c) Spatial distribution of holes in the $4 \mathrm{H}-\mathrm{SiC} \mathrm{p}-\mathrm{i}-\mathrm{n}$ diode for different activation energies of acceptors in the p-type injection layer at an injection current density of $200 \mathrm{~A} / \mathrm{cm}^{2}$. The hole density is normalized to the density of holes in the p-type injection layer. (d) Variation of the spatial distribution of holes in the $4 \mathrm{H}-\mathrm{SiC}$ p-i-n diode with the activation energy of acceptors in the p-type injection layer at an injection current density of $200 \mathrm{~A} / \mathrm{cm}^{2}$. The hole density is normalized to the density of holes in the p-type injection layer.
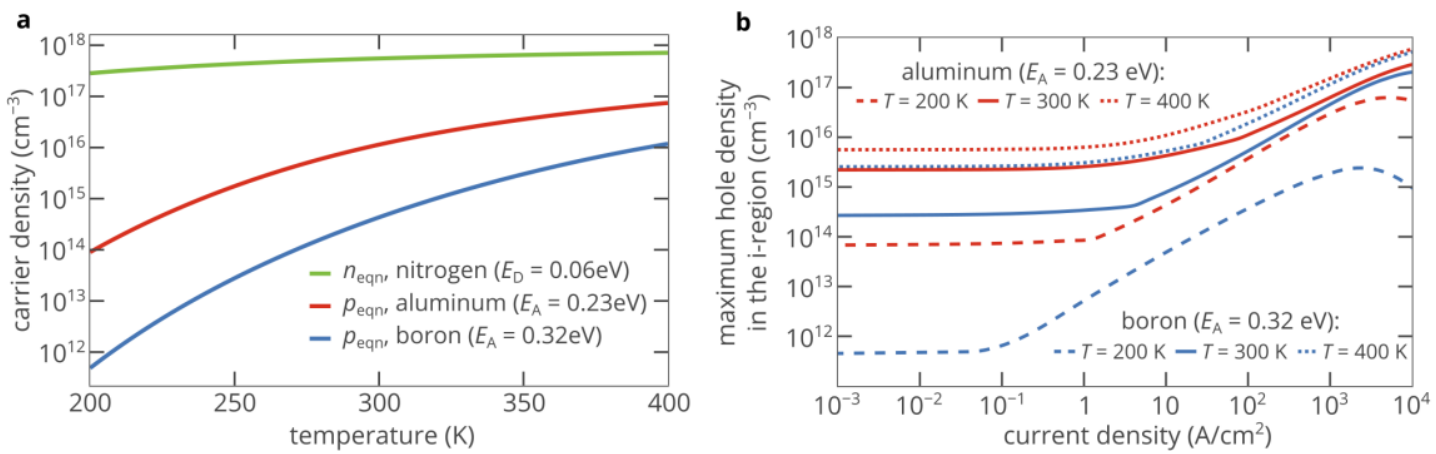

Figure 5. (a) Electron density in the n-type layer and hole density in the p-type layer as functions of temperature. (b) Dependence of the maximum hole density in the i-region of the $4 \mathrm{H}-\mathrm{SiC} \mathrm{p}-\mathrm{i}-\mathrm{n}$ diode on the injection current for three different temperatures and two different activation energies of acceptors. The $50 \mathrm{~nm}$-thick areas near the $\mathrm{p}-\mathrm{i}$ and $\mathrm{i}-\mathrm{n}$ junctions are ignored to avoid overestimation of the density of injected holes. The material parameters of $4 \mathrm{H}-\mathrm{SiC}$ at $200 \mathrm{~K}$ and $400 \mathrm{~K}$ are provided in the Supplementary Material. 


\subsection{Impact of Temperature}

Equations (6) and (7) show that the densities of free carriers in the p-type and n-type injection layers greatly depend on the device temperature. Since the activation energy of acceptors is very high, even a small decrease in temperature significantly decreases the hole density. If the p-type layer of the diode is doped with aluminum, the density of holes decreases by a factor of 160 as the temperature drops from $300 \mathrm{~K}$ to $200 \mathrm{~K}$ (Figure 5a). In the boron-doped p-type layer, the reduction in the hole density is even stronger: $p_{\text {eqp }}$ drops by a factor of 880 . However, this decrease in the free carrier density increases the asymmetry in the conduction properties of the n-type and p-type injection layer, which is beneficial for the superinjection effect, as discussed in the previous section. Figure $5 \mathrm{~b}$ shows that although at $T=200 \mathrm{~K}$, $p_{\text {eqp }}$ is lower by 160 than when $T=300 \mathrm{~K}$, the density of injected holes is only half that at room temperature at current densities above $300 \mathrm{~A} / \mathrm{cm}^{2}$. On the contrary, at high temperatures, the density of holes in the p-type layer is higher, and the superinjection effect is weaker, i.e., the ratio of the maximum injected hole density to the density of holes in the p-type layer is significantly lower than at room temperature.

\section{5. $6 \mathrm{H}$-SiC and 3C-SiC p-i-n Diodes}

Activation energies of acceptors and other material parameters in $6 \mathrm{H}-\mathrm{SiC}$ are roughly the same as in $4 \mathrm{H}-\mathrm{SiC}$ [30]. Accordingly, the strength of the superinjection effect and its other properties are very similar to that in $4 \mathrm{H}-\mathrm{SiC}$ p-i-n diodes. Since $4 \mathrm{H}-\mathrm{SiC}$ p-i-n diodes are already discussed, in this section, we focus only on $3 \mathrm{C}-\mathrm{SiC}$.

The activation energies of acceptors in $3 \mathrm{C}-\mathrm{SiC}$ are very similar to those in $4 \mathrm{H}-\mathrm{SiC}: 0.26 \mathrm{eV}$ for $\mathrm{Al}$ [35] and $0.34 \mathrm{eV}$ for $\mathrm{Ga}$ [35]. The electron and hole mobilities are slightly lower than in $4 \mathrm{H}-\mathrm{SiC}[36,37]$ and the carrier lifetime is slightly longer [38,39]. Equation (5) shows that the superinjection effect is not very sensitive to the carrier mobility, but inversely proportional to the carrier lifetime. Therefore, the hole injection in the 3C-SiC p-i-n diode is expected to be better than in the $4 \mathrm{H}-\mathrm{SiC}$ diode considered above, which agrees with the results of the self-consistent numerical simulations of the $3 \mathrm{C}-\mathrm{SiC} \mathrm{p}-\mathrm{i}-\mathrm{n}$ diode (see Figure 6).

\subsection{ZnS and AlN Diodes}

Although the activation energies of acceptors in silicon carbide are high, which limits the density of holes in the p-type material to $10^{14}-10^{15} \mathrm{~cm}^{-3}$, the p-type doping problem is even more pronounced in such materials as gallium nitride, aluminum nitride, and zinc sulfide. If in the case of GaN (which also features very high activation energies of acceptors [40]), one can use AlGaN/GaN/AlGaN heterostructures [41], the extremely high bandgap energy of AlN of $6.2 \mathrm{eV}$ simply excludes this option. At the same time, the activation energy of the most shallow acceptor in AlN is about $0.6 \mathrm{eV}$, which limits the density of holes in the p-type material to $\sim 10^{10} \mathrm{~cm}^{-3}$ [26]. Therefore, it is crucially important to improve the hole injection efficiency in AlN homojunction electronic and optoelectronic devices. Figure $6 \mathrm{~b}$ and the blue curve in Figure $6 \mathrm{~d}$ show the results of the numerical simulations for an AlN p-i-n diode. For the parameter of AlN, see Table S1 in the Supplementary Material. Despite the activation energy of acceptors in AlN being much higher than in silicon carbide, the maximum strength of the superinjection effect is lower, which is explained by lower mobility, slightly shorter carrier lifetime, and lower density of electrons in the n-type layer due to the higher activation energy of donors in AlN $(0.25 \mathrm{eV}$ [42]). The latter factor is especially important since the density of electrons in the n-type layer limits the maximum voltage at which Equations (1) and (2) are valid and therefore the maximum value of the electric field in Equation (5). In addition, the spatial distribution of the density of injected holes is remarkably different (Figure $6 \mathrm{~b}$ ). Due to the low density of free carriers in the i-region of the diode even at high bias voltages, the electric field in the i-region is strong. The electrostatic potential at the $p-i$ junction is well above the electrostatic potential at the $i-n$ junction. Hence, the potential well in the i-region is narrow, and a significant improvement in the density of injected holes is achieved only in a 
relatively narrow region near the i-n junction. Nevertheless, owing to the superinjection effect, at high bias voltages, the density of injected holes can exceed the doping limit by two orders of magnitude.

Another example of a wide-bandgap semiconductor, which can hardly be used as an active layer of the double heterostructure, is zinc sulfide [27]. At the same time, $\mathrm{ZnS}$ has long been known as a phosphor material owing to the efficient luminescence of different point defects [43]. However, electrical excitation of these defects was always a problem due to the extremely high activation energy of acceptors ( 0.6 eV [44]), which limits the density of holes in the p-type layer to less than $10^{10} \mathrm{~cm}^{-3}$. Therefore, it was proposed not to use $\mathrm{p}-\mathrm{n}$ and $\mathrm{p}$-i-n structures and try to implement the hole injection mechanism in metal/insulator/semiconductor structures [45]. Figure $6 \mathrm{~b}$ and the red curve in Figure $6 \mathrm{~d}$ show the simulated efficiency of hole injection in the ZnS p-i-n diode. For the parameters of the diode, see Table S1 in the Supplementary Material. These figures show that the hole density in the i-region of the structure is lower than $6 \times 10^{9} \mathrm{~cm}^{-3}$ until the current density exceeds $0.02 \mathrm{~A} / \mathrm{cm}^{2}$. Above $0.02 \mathrm{~A} / \mathrm{cm}^{2}$, the maximum density of injected holes rapidly increases with the injection current and can reach $7.8 \times 10^{12} \mathrm{~cm}^{-3}$, which is three orders of magnitude higher than the hole density in the p-type layer. Figure $6 \mathrm{c}$ shows that the superinjection effect in $\mathrm{ZnS}$ is very similar to that in AlN. However, its maximum strength is higher (mostly due to the higher density of electrons in the n-type layer), and the effect can be observed at lower currents due to the longer carrier lifetimes (see Table S1 in the Supplementary Material).
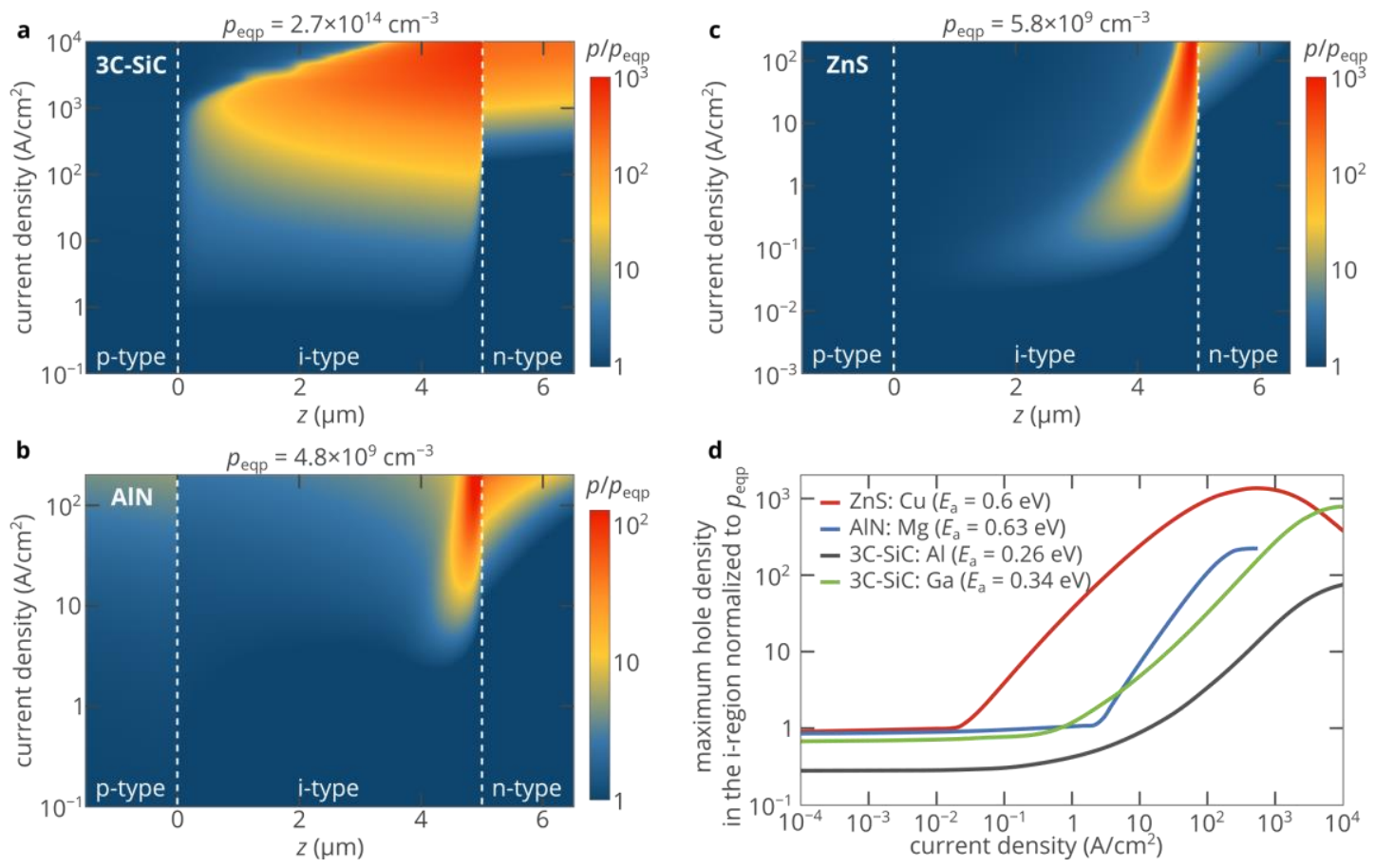

Figure 6. $(\mathbf{a}-\mathbf{c})$ Spatial distribution of the hole density in the i-region of the three $p-i-n$ diodes based on $3 \mathrm{C}-\mathrm{SiC}$ with the p-layer doped with gallium (a), AlN (b), and ZnS (c). The hole density is normalized to the hole density in the p-type layer. (d) Dependence of the maximum hole density in the i-region of different the $\mathrm{p}-\mathrm{i}-\mathrm{n}$ diodes. The $50 \mathrm{~nm}$-thick areas near the $\mathrm{p}-\mathrm{i}$ and $\mathrm{i}-\mathrm{n}$ junctions are ignored to avoid overestimation of the density of injected holes. The hole density is normalized to the density of holes in the p-type injection layer. For the $3 \mathrm{C}$-SiC diode, two dopants ( $\mathrm{Al}$ and $\mathrm{Ga}$ ) of the p-type layer are considered. The parameters of the $3 \mathrm{C}-\mathrm{SiC}, \mathrm{ZnS}$, and AlN diodes are listed in the Supplementary Material.

\section{Conclusions}

We have numerically demonstrated the superinjection of holes in homojunction p-i-n diodes based on different wide-bandgap semiconductors. This effect gives the possibility to create a high density of nonequilibrium holes in the i-region of the $\mathrm{p}$-i-n diode at high forward bias voltages. 
The created density of holes exceeds the hole density in the p-type injection layer by more than two orders of magnitude, which can be exploited to improve the performance of light-emitting devices. The highest hole density is found near the $\mathrm{i}-\mathrm{n}$ junction, which contradicts the common belief about $\mathrm{p}$-i-n diodes, since in the i-region, one could expect to find the highest hole density near the $\mathrm{p}$-i junction. However, we have provided a clear physical explanation for the observed effect, which is based on the asymmetry in the free carrier densities in the n-type and p-type layers. In $\mathrm{SiC}, \mathrm{GaN}, \mathrm{AlN}, \mathrm{ZnS}$, and many other wide-bandgap semiconductors, this asymmetry is naturally provided by the high activation energy of acceptors, which greatly limits the density of holes in the p-type layer of the diode. Hence, for optoelectronic devices, it is crucially important to overcome this doping limit, especially if it is not possible to use heterostructures. We have demonstrated that the superinjection of holes can be observed at high forward bias voltages. At the same time, the superinjection effect does not show itself at low and moderate injection currents (bias voltages). The higher the density of holes in the p-type layer, the higher the threshold current (see Figure 4b). This is one of the reasons why the effect was not reported previously.

The strength of the superinjection effect depends on the minority carrier lifetime and other properties of the semiconductor diode, but the most crucial parameter is the activation energy of acceptors, $E_{\mathrm{A}}$, as it determines the hole density in the p-type region. We found that the superinjection of holes can hardly be detected at doping levels above $10^{17} \mathrm{~cm}^{-3}$ if $E_{\mathrm{A}}$ is lower than $\sim 0.15 \mathrm{eV}$. On the other hand, at high activation energies $\left(E_{\mathrm{A}} \gtrsim 0.4 \mathrm{eV}\right)$, it is strong even at relatively low injection currents. Assuming the density of electrons to be high in the n-type region of the p-i-n diode $\left(n_{\text {eqn }} \gtrsim 10^{17} \mathrm{~cm}^{-3}\right)$, the results of the numerical simulations and Equation (5) show that the higher the activation energy of acceptors, the stronger the effect. However, we should emphasize that the absolute value of the density of injected holes in a diode with a high activation energy of acceptors in the p-type layer (e.g., $E_{\mathrm{A}}=0.32 \mathrm{eV}$ ) cannot exceed the absolute value of the density of injected holes in a diode with a low activation energy of acceptors in the p-type layer (e.g., $E_{\mathrm{A}}=0.23 \mathrm{eV}$ ) (see Figure 5). Therefore, the key to the improvement of the hole injection efficiency is a low activation energy of acceptors. However, if $E_{\mathrm{A}}$ cannot be reduced, the superinjection effect is the only possible means to improve the hole injection efficiency in homojunction diodes.

To directly measure the theoretically predicted strength of the superinjection effect, one should study two single color centers $[6,8,14,24,25,46,47]$ in the i-region of the p-i-n diode under a high forward bias voltage: one near the $\mathrm{p}-\mathrm{i}$ junction and one near the $\mathrm{i}-\mathrm{n}$ junction. Since the single-photon electroluminescence process of the color center is determined by the electron and hole capture processes $[8,24]$, whose rates are directly proportional to the electron and hole densities in the vicinity of the color center, the ratio of the single-photon emission rates of these two color centers can give the information about the ratio of the hole densities at the positions of the color centers, and therefore about the strength of the superinjection effect.

A disadvantage of the superinjection effect is that when a very high density of injected holes is achieved, the electron current is at least an order of magnitude higher than the hole current. Therefore, the energy efficiency of LEDs based on the superinjection effect in homojunction p-i-n diodes will be lower than that of those based on double heterostructures. A strong effect also requires high bias voltages ( $>6 \mathrm{~V}$ for $4 \mathrm{H}-\mathrm{SiC}$ diodes and $>30 \mathrm{~V}$ for AlN) that can create a high electric field in the p-type region of the diode (see Equation (5)). Accordingly, the heat generation rate is considerably high: more than $100 \mathrm{~W} / \mathrm{cm}^{2}$ for the $\mathrm{ZnS}$ diode and more than $\sim 1 \mathrm{~kW} / \mathrm{cm}^{2}$ for other considered diodes. This should also be taken into account in practical applications.

We should emphasize that the superinjection effect not only gives a unique possibility to create a density of nonequilibrium holes which is orders of magnitude higher than the doping limit $p_{\text {eqp }}$, but also provides it in the vicinity of the $\mathrm{i}-\mathrm{n}$ junction, where the density of injected electrons is maximal (see Figure 2c,d). Thus, we obtain the maximum electron and hole densities at roughly the same place. This feature is extremely important [24] for the design and development of ultrabright electrically driven single-photon sources based on point defects in $\mathrm{SiC}, \mathrm{AlN}, \mathrm{ZnO}$, and other 
wide-band gap semiconductors $[6,24,25,28,46-48]$, which are key elements for many applications of quantum information science, such as quantum-communication networks and optical quantum computers $[49,50]$. We believe that our findings will stimulate research focused on the development of novel high-performance light sources based on wide-bandgap semiconductors.

Supplementary Materials: The following are available online at http://www.mdpi.com/1996-1944/12/12/1972/s1, Table S1: Main material parameters used in the numerical simulations, Table S2: Temperature dependence of the main material parameters of $4 \mathrm{H}-\mathrm{SiC}$, Figure S1: Comparison of the theory and numerical simulations.

Author Contributions: I.A.K. performed the numerical simulations. D.Y.F. conceived the idea and wrote the manuscript. Both authors analyzed the results.

Funding: The work is supported by the Russian Science Foundation (17-79-20421).

Conflicts of Interest: The authors declare no conflict of interest.

\section{References}

1. Schubert, E.F. Light-Emitting Diodes; Cambridge University Press: Cambridge, UK, 2006.

2. Casey, H.C., Jr.; Panish, M.B. Heterostructure Lasers; Academic Press: New York, NY, USA, 2012.

3. Agrawal, G.P.; Dutta, N.K. Semiconductor Lasers; Springer: New York, NY, USA, 2013.

4. Witzens, J. High-Speed Silicon Photonics Modulators. Proc. IEEE 2018, 106, 2158-2182. [CrossRef]

5. Peng, Z.; Fattal, D.; Fiorentino, M.; Beausoleil, R. CMOS-Compatible Microring Modulators for Nanophotonic Interconnect. In Integrated Photonics Research, Silicon and Nanophotonics and Photonics in Switching; Optical Society of America: Washington, DC, USA, 2010.

6. Lohrmann, A.; Iwamoto, N.; Bodrog, Z.; Castelletto, S.; Ohshima, T.; Karle, T.J.; Gali, A.; Prawer, S.; McCallum, J.C.; Johnson, B.C. Single-photon emitting diode in silicon carbide. Nat. Commun. 2015, 6, 7783. [CrossRef] [PubMed]

7. Yuan, Z.; Kardynal, B.E.; Stevenson, R.M.; Shields, A.J.; Lobo, C.J.; Cooper, K.; Beattie, N.S.; Ritchie, D.A.; Pepper, M. Electrically driven single-photon source. Science 2002, 295, 102-105. [CrossRef]

8. Fedyanin, D.Y.; Agio, M. Ultrabright single-photon source on diamond with electrical pumping at room and high temperatures. New J. Phys. 2016, 18, 073012. [CrossRef]

9. Piprek, J. Semiconductor Optoelectronic Devices: Introduction to Physics and Simulation; Academic Press: New York, NY, USA, 2013.

10. Kasap, S.; Capper, P. Springer Handbook of Electronic and Photonic Materials; Springer: New York, NY, USA, 2017.

11. Zhang, S.B.; Wei, S.-H.; Zunger, A. A phenomenological model for systematization and prediction of doping limits in II-VI and I-III-VI2 compounds. J. Appl. Phys. 1998, 83, 3192-3196. [CrossRef]

12. Yan, Y.; Wei, S.-H. Doping asymmetry in wide-bandgap semiconductors: Origins and solutions. Phys. Status Solidi (b) 2008, 245, 641-652. [CrossRef]

13. Traoré, A.; Koizumi, S.; Pernot, J. Effect of n- and p-type doping concentrations and compensation on the electrical properties of semiconducting diamond. Phys. Status Solidi (a) 2016, 213, 2036-2043. [CrossRef]

14. Khramtsov, I.A.; Fedyanin, D.Y. Superinjection in diamond p-i-n diodes: Bright single-photon electroluminescence of color centers beyond the doping limit. arXiv 2018, arXiv:1804.01066.

15. Neumark, G.F. Wide bandgap light-emitting devices materials and doping problems. Mater. Lett. 1997, 30, 131-135. [CrossRef]

16. Park, J.H.; Kim, D.Y.; Fred Schubert, E.; Cho, J.; Kim, J.K. Fundamental Limitations of Wide-Bandgap Semiconductors for Light-Emitting Diodes. ACS Energy Lett. 2018, 3, 655-662. [CrossRef]

17. Alferov, Z.I.; Khalfin, V.B.; Kazarinov, R.F. A characteristic feature of injection into heterojunctions. Sov. Phys. Solid State 1967, 8, 2480.

18. Kazarinov, R.F.; Suris, R.A. Superinjection of carriers in variable-gap pn structures. Sov. Phys. Semicond. 1975, 9, 6-10.

19. Piprek, J. Nitride Semiconductor Devices: Principles and Simulation; Wiley: Weinheim, Germany, 2007.

20. Morkoç, H. Handbook of Nitride Semiconductors and Devices, Materials Properties, Physics and Growth; Wiley: Weinheim, Germany, 2009.

21. Alferov, Z.I. Nobel Lecture: The double heterostructure concept and its applications in physics, electronics, and technology. Rev. Mod. Phys. 2001, 73, 767-782. [CrossRef] 
22. Khramtsov, I.A.; Fedyanin, D.Y. Superinjection in single-photon emitting diamond diodes. In Proceedings of the 2018 International Conference on Numerical Simulation of Optoelectronic Devices (NUSOD), Hong Kong, China, 5-9 November 2018; pp. 123-124. [CrossRef]

23. Khramtsov, I.A.; Fedyanin, D.Y. Superinjection in diamond homojunction P-I-N diodes. Semicond. Sci. Technol. 2019, 34, 03LT03. [CrossRef]

24. Khramtsov, I.A.; Vyshnevyy, A.A.; Fedyanin, D.Y. Enhancing the brightness of electrically driven single-photon sources using color centers in silicon carbide. npj Quantum Inf. 2018, 4, 15. [CrossRef]

25. Widmann, M.; Niethammer, M.; Makino, T.; Rendler, T.; Lasse, S.; Ohshima, T.; Hassan, J.U.; Son, N.T.; Lee, S.-Y.; Wrachtrup, J. Bright single photon sources in lateral silicon carbide light emitting diodes. Appl. Phys. Lett. 2018, 112, 231103. [CrossRef]

26. Taniyasu, Y.; Kasu, M.; Makimoto, T. An aluminium nitride light-emitting diode with a wavelength of 210 nanometres. Nature 2006, 441, 325-328. [CrossRef]

27. Xu, X.; Li, S.; Chen, J.; Cai, S.; Long, Z.; Fang, X. Design Principles and Material Engineering of ZnS for Optoelectronic Devices and Catalysis. Adv. Funct. Mater. 2018, 28, 1802029. [CrossRef]

28. Lienhard, B.; Lu, T.-J.; Jeong, K.-Y.; Moon, H.; Iranmanesh, A.; Grosso, G.; Englund, D. High-purity single photon emitter in aluminum nitride photonic integrated circuit. In Proceedings of the 2017 Conference on Lasers and Electro-Optics Europe \& European Quantum Electronics Conference (CLEO/Europe-EQEC), Munich, Germany, 25-29 June 2017.

29. Evwaraye, A.O.; Smith, S.R.; Mitchel, W.C. Shallow and deep levels in n-type 4H-SiC. J. Appl. Phys. 1996, 79, 7726-7730. [CrossRef]

30. Shur, M.; Rumyantsev, S.; Levinshtein, M. Sic Materials and Devices; World Scientific: Singapore, 2006.

31. Pernot, J.; Zawadzki, W.; Contreras, S.; Robert, J.L.; Neyret, E.; Di Cioccio, L. Electrical transport in n-type 4H silicon carbide. J. Appl. Phys. 2001, 90, 1869-1878. [CrossRef]

32. Fedyanin, D.Y.; Arsenin, A.V. Surface plasmon polariton amplification in metal-semiconductor structures. Opt. Express 2011, 19, 12524-12531. [CrossRef] [PubMed]

33. Vyshnevyy, A.A.; Fedyanin, D.Y. Self-Heating and Cooling of Active Plasmonic Waveguides. ACS Photonics 2016, 3, 51-57. [CrossRef]

34. Sze, S.M. Physics of Semiconductor Devices; Wiley: Weinheim, Germany, 1981.

35. Harris, G.L. Properties of Silicon Carbide; IET: London, UK, 1995.

36. Roschke, M.; Schwierz, F. Electron mobility models for $4 \mathrm{H}, 6 \mathrm{H}$, and 3C SiC [MESFETs]. IEEE Trans. Electron Devices 2001, 48, 1442-1447. [CrossRef]

37. Lophitis, N.; Arvanitopoulos, A.; Perkins, S.; Antoniou, M. TCAD Device Modelling and Simulation of Wide Bandgap Power Semiconductors. In Disruptive Wide Bandgap Semiconductors, Related Technologies, and Their Applications; Sharma, Y.K., Ed.; InTechOpen: London, UK, 2018.

38. Grivickas, V.; Manolis, G.; Gulbinas, K.; Jarašiūnas, K.; Kato, M. Excess carrier recombination lifetime of bulk n-type 3C-SiC. Appl. Phys. Lett. 2009, 95, 242110. [CrossRef]

39. Arvanitopoulos, A.; Lophitis, N.; Gyftakis, K.N.; Perkins, S.; Antoniou, M. Validated physical models and parameters of bulk 3C-SiC aiming for credible technology computer aided design (TCAD) simulation. Semicond. Sci. Technol. 2017, 32, 104009. [CrossRef]

40. Brochen, S.; Brault, J.; Chenot, S.; Dussaigne, A.; Leroux, M.; Damilano, B. Dependence of the Mg-related acceptor ionization energy with the acceptor concentration in p-type GaN layers grown by molecular beam epitaxy. Appl. Phys. Lett. 2013, 103, 032102. [CrossRef]

41. Cheng, K.; Liang, H.; Van Hove, M.; Geens, K.; De Jaeger, B.; Srivastava, P.; Kang, X.; Favia, P.; Bender, H.; Decoutere, S.; et al. AlGaN/GaN/AlGaN Double Heterostructures Grown on 200 mm Silicon (111) Substrates with High Electron Mobility. Appl. Phys. Express 2012, 5, 011002. [CrossRef]

42. Taniyasu, Y.; Kasu, M.; Makimoto, T. Electrical conduction properties of $n$-type Si-doped AlN with high electron mobility $\left(>100 \mathrm{~cm}^{2} \mathrm{~V}^{-1} \mathrm{~s}^{-1}\right)$. Appl. Phys. Lett. 2004, 85, 4672-4674. [CrossRef]

43. McCloy, J.S.; Potter, B.G. Photoluminescence in Chemical Vapor Deposited ZnS: Insight into electronic defects. Opt. Mater. Express 2013, 3, 1273-1278. [CrossRef]

44. Bube, R.H. Photoconductivity of Solids; Wiley: New York, NY, USA, 1978.

45. Ohno, T.; Kurisu, K.; Taguchi, T. Growth of high-quality cubic ZnS crystals and their application to MIS blue light-emitting diodes. J. Cryst. Growth 1990, 99, 737-742. [CrossRef] 
46. Berhane, A.M.; Jeong, K.-Y.; Bodrog, Z.; Fiedler, S.; Schröder, T.; Triviño, N.V.; Palacios, T.; Gali, A.; Toth, M.; Englund, D.; et al. Bright Room-Temperature Single-Photon Emission from Defects in Gallium Nitride. Adv. Mater. 2017, 29, 1605092. [CrossRef] [PubMed]

47. Choi, S.; Berhane, A.M.; Gentle, A.; Ton-That, C.; Phillips, M.R.; Aharonovich, I. Electroluminescence from localized defects in zinc oxide: Toward electrically driven single photon sources at room temperature. ACS Appl. Mater. Interfaces 2015, 7, 5619-5623. [CrossRef] [PubMed]

48. Wang, J.; Zhou, Y.; Wang, Z.; Rasmita, A.; Yang, J.; Li, X.; von Bardeleben, H.J.; Gao, W. Bright room temperature single photon source at telecom range in cubic silicon carbide. Nat. Commun. 2018, 9, 4106. [CrossRef] [PubMed]

49. Boretti, A.; Rosa, L.; Mackie, A.; Castelletto, S. Electrically Driven Quantum Light Sources. Adv. Opt. Mater. 2015, 3, 1012-1033. [CrossRef]

50. Aharonovich, I.; Englund, D.; Toth, M. Solid-state single-photon emitters. Nat. Photonics 2016, 10, 631-641. [CrossRef]

C 2019 by the authors. Licensee MDPI, Basel, Switzerland. This article is an open access article distributed under the terms and conditions of the Creative Commons Attribution (CC BY) license (http://creativecommons.org/licenses/by/4.0/). 